\title{
Simultaneous Supplies of Dirty Energy and Capacity Constrained Clean Energy: Is There a Green Paradox?
}

\author{
Marc Gronwald $^{1}$ • Ngo Van Long ${ }^{2,3}$ - Luise Roepke ${ }^{4}$
}

Accepted: 5 April 2017 / Published online: 5 July 2017

(C) The Author(s) 2017. This article is an open access publication

\begin{abstract}
The effects of two popular second-best clean energy policies are analysed using an extended Hotelling-type resource extraction framework. This model features, first, heterogenous energy sources and, second, a capacity-constrained backstop technology. This setup allows for capturing the following two empirical observations. First, different types of energy sources are used simultaneously despite different production cost. Second, experiences from various European countries show that a further expansion of the use of climate friendly technologies faces substantial technological as well as political constraints. We use this framework to analyse if under two policy scenarios a so-called "Green Paradox" occurs. A subsidy for the clean energy as well as an expansion of the capacity of the clean energy are considered. The analysis shows that under plausible parameter values both policy measures lead to a weak Green Paradox; however a strong Green Paradox is only found for the capacity expansion scenario. In addition, the subsidy is found to be welfare enhancing while the capacity increase is welfare enhancing only if the cost of adding the capacity is sufficiently
\end{abstract}

Marc Gronwald and Luise Roepke gratefully acknowledge financial support by the German Federal Ministry of Education and Research. The authors are indebted to the editors and two anonymous reviewers for their very helpful comments and guidance.

Electronic supplementary material The online version of this article (doi:10.1007/s10640-017-0151-6) contains supplementary material, which is available to authorized users.

$凶 \quad$ Marc Gronwald

mgronwald@abdn.ac.uk

Ngo Van Long

ngo.long@mcgill.ca

Luise Roepke

roepke@ifo.de

1 CESifo and ifo Institute, University of Aberdeen Business School, Aberdeen AB24 3QY, UK

2 Department of Economics, McGill University, Montreal H3A 2T7, Canada

3 University of Tasmania, Hobart, Australia

4 ifo Institute for Economic Research, Munich, Germany 
small.We also show the effects of the policies crucially depend on the initial capacity and that under certain scenarios even an "extreme" Green Paradox is found.

Keywords Capacity constraints - Green Paradox - Climate change - Simultaneous resource use $\cdot$ Cost reversal

JEL Classification Q38 $\cdot \mathrm{Q} 54 \cdot \mathrm{H} 23$

\section{Introduction}

The decarbonisation of the global economy is very high on the global political agenda. As various types of clean technologies are available, the situation looks generally promising: Wind as well as solar energy generally could replace conventional fossil fuel power plants; thus, electricity generation potentially could become considerably cleaner. The situation in the transport sector is similar: biofuels have the potential to replace conventional fuels. What is more, a considerable political will is evident, and has manifested itself in various types of policy measures such as feed-in tariffs for renewable energy or biofuel mandates.

However, this decarbonisation process necessarily involves nothing short of an entire reconstruction of the global energy sector. Thus, it is clearly not an easy task. It is not just that this process is of large scale and involves complex investment projects with very long horizons, it also seems to meet increasing resistance in the population. To name just a few examples, land used for production of biofuels reduces the land areas for growing food. This results in concerns about food security and sustainability. As long as more advanced technologies such as second generation biofuels are not yet available, the situation looks difficult. On the electricity generation front, the situation is similar: in countries such as Germany and the United Kingdom, the installation of additional wind generation capacities becomes increasingly difficult as it finds insufficient support in many local communities. An additional challenge in this context is the installation of enormous amounts of electricity transmission capacities - this is often equally unwelcome. Greatly improved energy storage technologies would certainly be very helpful in this regard but are not available yet.

The consequence of these challenges is that while clean technologies are certainly used in various countries, in particular in Europe, the available capacities are not sufficient for meeting the complete energy demand and, in addition, expanding the use of clean energies is getting increasingly complicated. This constitutes a major explanation of two empirical facts. First, both fossil energy and clean energy are produced simultaneously even though the latter is still considerably more expensive than the former. Second, climate friendly energy is used but its capacity is severely constrained. Further expansions are challenging because of technological and/or political constraints. To capture these two empirical observations, our paper proposes a resource extraction model with an exhaustible resource stock in competition with a capacity-constrained clean backstop. ${ }^{1}$

This framework is then used to analyse two different scenarios. First, assuming that the first-best carbon tax is not politically feasible, we consider the introduction of a subsidy on the clean technology. Subsidizing the clean energy sector is a very common and popular second-best policy measure. Second, the effect of an expansion of the capacity-an increase

1 In a working paper version (Grondwald et al. 2016), we modify the multi-deposit model of Holland (2003) and consider the case with several heterogeneous dirty resources; this reflects the simultaneous use of e.g. both conventional and unconventional oil. The results are broadly similar, except there is a jump in emissions when the more dirty oil replaces the conventional oil. 
in the availability - of the backstop is analysed. This can be more broadly interpreted, to include the sudden availability of a new technology which allows using clean technologies to a much larger extent, e.g. a breakthrough in areas such as advanced biofuels or energy storage. The latter would allow a massive increase in the use of renewable electricity. Technological breakthroughs of this type may be the result of a public policy such as research and development subsidies. The effects on the extraction path of the dirty exhaustible resource and on the total welfare are analysed. Specifically, we ask if there are negative consequences for the climate when second-best policies are implemented. As Sinn (2008) puts it: is there a Green Paradox? The analysis conducted in this paper involves both analytical and numerical parts; the calibration of the numerical part is based on empirical data on the global crude oil market. The analysis, finally, employs the notions of a "weak Green Paradox" and a "strong Green Paradox" introduced by Gerlagh (2011). The former describes a short-term increase of anthropogenic emissions in response to a policy measure, the latter an increase in cumulative damages. In addition, we also introduce the concept of an extreme Green Paradox: this captures a decrease in welfare caused by well-intentioned but poorly designed climate policy measures.

Our numerical analysis shows that, under our base-line specification of parameter values, whereas both policy measures lead to a weak Green Paradox, a strong Green Paradox is only found for the capacity expansion scenario. In addition, the subsidy is found to be welfare enhancing while the capacity increase is welfare enhancing only if the cost of adding the capacity is sufficiently small. In terms of the present value of the stream of damage costs, we find that a subsidy of $25 \%$ on the clean energy will reduce total damage costs by about $6 \%$, while a capacity expansion of $20 \%$ will increase total damage costs by about $2.5 \%$. The reason is that a subsidy makes clean energy production profitable at an earlier date, resulting in pushing the fossil resource exhaustion date further into the future, so that the pollution stock peaks at a later date. In contrast, a capacity expansion reduces the maximum price that the last drop of oil would earn, and has a negative effect on the residual demand for oil. These effects give a strong incentive for fossil resource owners to pump more oil out in the earlier stage. A sensitivity analysis we conduct shows that an extreme Green Paradox-a decrease in welfare-occurs when the damages associated with carbon emissions are sufficiently high. In addition, we also find that the effects crucially depend on the initial size of the capacity.

Assuming that the climate friendly backstop technology is capacity constrained makes a significant contribution to the Green Paradox literature. ${ }^{2}$ Up to now, all papers which contain a backstop technology assume that at some point a backstop technology becomes (economically) available in unlimited amounts and replaces conventional energy sources completely. ${ }^{3}$ Our assumption of a capacity constrained backstop technology allows for the analysis of a completely new scenario: what are the consequences of an increase in the availability of clean energy? Hoel (2011) contributes to the "traditional" backstop technology using a two-country model. His analysis shows that the degree of country heterogeneity has significant effects on how subsidies or taxes on the one hand and emissions paths on the other are related. van der Ploeg and Withagen (2012a, b) offer an interesting refinement: they show that the cost of a backstop technology are essential for the existence or non-existence of a Green Paradox outcome. If the backstop is relatively expensive and, thus, full exhaustion of the non-renewable resource is optimal, a Green Paradox occurs. However, if the backstop

\footnotetext{
2 van der Ploeg and Withagen (2015) as well as Jensen et al. (2015) provide excellent overviews of the Green Paradox literature.

3 The more the capacity constraint is relaxed, the more the clean substitute becomes a "classic" backstop technology. We model the backstop technology in line with Dasgupta and Heal (1974), as a "perfectly durable commodity, which provides a flow of services at constant rate."
} 
is sufficiently cheap this finding is reversed. Hoel and Jensen's (2012) paper also consider different types of climate friendly technologies and show e.g. that carbon capture and storage can have different effects than renewable energies. Michielsen (2014), in contrast, considers a more refined dirty resource sector. His paper shows that, under certain conditions, the anticipation of a climate policy can actually reduce current emissions: a so-called Green Orthodox occurs. A key factor of his model is the degree of substitutability between the dirty resources. Grafton et al. (2012) analyse the effects of biofuel subsidies. Their paper shows that whether or not a Green Paradox occurs depends on factors such as the extraction cost of the fossil resource and/or marginal cost of using biofuels.

The remainder of the paper is organised as follows. In the next section, we derive a model of substitute production under a capacity constraint. Section 3 describes the first-best solution; Sects. 4 and 5 discuss two feasible policy scenarios and a sensitivity analysis. Section 6 illustrates the policy relevance of this paper. Section 7 offers some concluding remarks.

\section{A Model of Substitute Production Under Capacity Constraint}

Assume that there is an aggregate stock of fossil fuels, denoted by $S$. The constant per unit extraction cost is denoted by $c$. There is no capacity constraint on the amount of extraction at any given point of time $t$. The cumulative extraction constraint is

$$
\int_{0}^{\infty} q(t) d t \leq S(0) .
$$

The rate of extraction is denoted by $q$. Then we have $\dot{S}(t)=-q(t)$, with $S(0)=S_{0}$. Following van der Ploeg and Withagen (2012a, b), we assume a pollution decay rate of zero. Then the stock of pollution, denoted by $X$, evolves according to the rule $\dot{X}=\eta q$, with $X(0)=X_{0}$, given. Here $\eta$ is the pollution content per unit. The maximum possible stock of pollution is $\bar{X}$, where $\bar{X}=X_{0}+\eta S_{0}$. The damage cost at time $t$ depends on the stock $X(t)$. The damage function is denoted by $G(X)$. We assume that $G^{\prime}(X)>0$ and $G^{\prime \prime}(X) \geq 0$.

There is a clean energy that is a perfect substitute for the fossil fuels. Let $q_{g}(t)$ be the amount of clean energy produced at time $t$. The key contribution of this paper is the assumption that there is a capacity constraint on clean energy production: $q_{g}(t) \leq \bar{q}_{g}$, where the subscript $g$ is used for the clean (or 'green') energy. This means that at each point of time, the amount of clean energy that can be produced is exogenously determined by the capacity constraint. Let $c_{g}$ be the constant unit cost of production of the clean energy. Assume $c_{g}>c$.

Let $Q(t)=q(t)+q_{g}(t)$ denote the aggregate supply of energy at time $t$, where some of these outputs may be zero. The utility of consuming $Q(t)$ is $U[Q(t)]$, where $U(\cdot)$ is a strictly concave and increasing function and $U^{\prime}(0)$ can be finite or infinite. The instantaneous welfare at time $t$ is

$$
W(t)=U(Q(t))-c q(t)-c_{g} q_{g}(t)-G(X(t))
$$

Consumer' demand is represented by the condition $p=U^{\prime}(Q)$. Inverting this function, we obtain the demand function $Q=D(p), D^{\prime}(p)<0$. We assume that when the price of energy is equal to $c_{g}$, the market demand for energy exceeds the capacity $\bar{q}_{g}$. We denote by $\bar{p}$ the consumer's marginal utility when energy consumption is at the green capacity level, $\bar{q}_{g}$, i.e. $D(\bar{p})=\bar{q}_{g}$.

Our first task is to characterize the equilibrium in the perfect competition situation, in the absence of a carbon tax. The resource owners follow a Hotelling-type extraction path, 
maximizing the value of the resource stock such that the resource rent increases at the rate of interest. Since the renewable resource owners do not have to optimise intertemporarily, their supply behaviour is different from that of the exhaustible resource owners. In the next subsection we impose a condition which ensures that the green energy is not produced during some initial phase.

\subsection{Extraction in the Absence of a Carbon Tax}

We will consider a scenario with a subsidy rate $s \geq 0$ per unit of clean energy, and restrict $s$ to belong to some closed interval $[0, \bar{s}]$, where $\bar{s}$ is the maximum subsidy that is politically feasible. The clean energy firms's private unit cost is defined as its unit production cost minus the subsidy, $c_{g}^{\#}(s)=c_{g}-s$. We assume that $c_{g}^{\#}(s)>c$, for all politically feasible $s \in[0, \bar{s}]$. Thus the marginal cost of extracting the fossil resource is lower than the clean energy firms' private unit cost. When $p(t)$ reaches $c_{g}^{\#}(s)$, demand must be met from both the clean energy sector and fossil fuel extraction. For all $p$ in the range $\left[c_{g}^{\#}(s), \bar{p}\right]$, the clean energy will always be produced at maximum capacity. Given the limited green capacity $\bar{q}_{g}$, the equilibrium price of energy can never exceed $\bar{p} \equiv U^{\prime}\left(\bar{q}_{g}\right)$.

Throughout most of the analysis, we assume that the initial size of the exhaustible resource stock, $S_{0}$, is large enough so that in the absence of a subsidy the clean energy is uncompetive during some initial time interval. Let us formalize this condition. Let $x$ denote the number of years it would take for the price $p$ to rise from $p=c_{g}$ to $p=\bar{p}$, i.e., $x=(1 / r)\left[\ln (\bar{p}-c)-\ln \left(c_{g}-c\right)\right]$. Let $t^{*}$ be the time at which the energy price reaches $c_{g}$ and $\bar{T}$ the time of resource exhaustion, i.e., $p(\bar{T})=\bar{p}$. We assume that $S_{0}$ is sufficiently large, such that

$$
S_{0}>\int_{t^{*}}^{\bar{T}} D(p(t)) d t-\left(\bar{T}-t_{g}\right) \bar{q}_{g} \equiv \int_{0}^{x} D\left[c+\left(c_{g}-c\right) e^{r \tau}\right] d \tau-x \bar{q}_{g} \equiv \widetilde{S}
$$

For ease of reference, we label the required condition as Condition 1.4

\subsection{Three Phases of Resource Utilization and the Price Path}

The equilibrium path of the energy price is continuous and the resource use pattern can be described as follows.

Phase 1: Energy is supplied only by extraction from the exhaustible resource stock. This phase ends at an endogenously determined time $t_{g}^{\#}>0$, such that the price at time $t_{g}^{\#}$ is equal to $c_{g}^{\#}(s)$. During this phase, the net price of the fossil resource, $p(t)-c$, rises at a rate equal to the interest rate $r .^{5}$

Phase 2: Energy is simultaneously supplied by extraction from the resource deposit $S$ and the (more costly) renewable energy running at its capacity level $\bar{q}_{g}$. This phase begins

4 When there is a subsidy $s \in[0, \bar{s}]$, there will still be an initial phase where the only energy source comes from the fossil fuels, if the following stronger condition, called Condition 1b, is satisfied:

$$
S_{0}>\widetilde{S}^{\#}(s) \equiv \int_{0}^{x^{\#}(s)} D\left[c+\left(c_{g}^{\#}(s)-c\right) e^{r \tau}\right] d \tau-x^{\#}(s) \bar{q}_{g}
$$

where $x^{\#}(s)$ is the number of years it would take for the price $p$ to rise from $p=c_{g}^{\#}(s)$ to $p=\bar{p}$, that is, $x^{\#}(s)=(1 / r)\left[\ln (\bar{p}-c)-\ln \left(c_{g}^{\#}(s)-c\right)\right]$.

5 Note that since $p\left(t^{*}\right)=c_{g}$, we have $t_{g}^{\#}<t^{*}$ if there is a positive subsidy $s$ per unit of clean energy. 
at time $t_{g}^{\#}$ and ends at an endogenously determined $\bar{T}$, at which time the resource stock $S$ is exhausted. During this phase the resource rent, $p(t)-c$, continues to rise at rate $r$.

Phase 3: The only source of energy is clean energy, available at capacity level $\bar{q}_{g}$. The price is constant at $\bar{p}$. This phase begins at time $\bar{T}$ and continues for ever.

At time $t_{g}^{\#}$ the clean energy sector's supply $\bar{q}_{g}$ is not enough to meet the demand $D\left(c_{g}^{\#}(s)\right)$. The shortfall, called residual demand, is met at time $t_{g}^{\#}$ by extraction from the exhaustible, such that $\bar{q}_{g}+q\left(t_{g}^{\#}\right)=D\left(c_{g}^{\#}(s)\right)$. From time $t_{g}^{\#}$ on, only the residual demand must be met by the exhaustible resource, indicating that the existence of a constrained renewable resource alleviates the scarcity problem of the exhaustible resources.

\subsection{Numerical Analysis}

In addition to theoretical analyses, this paper also offers a numerical illustration coupled with a sensitivity analysis. This section briefly summarises parameter choices of the based line scenario. The general aim is to capture relationships observable in the global crude oil market. We set $\bar{q}_{g}=5, r=0.01, c=1.25$, and $c_{g}=4$. Moreover, we assume linear demand, $D(p)=A-p$, where $A=20$. This implies $\bar{p}=15$. To compute the pollution stock, we specify the stock size $S_{0}$. We assume $S_{0}=2000$, which is an aggregation of conventional oil reserves and non-conventional oil reserves. ${ }^{6}$ The ratio between marginal costs of fossil fuel extraction and green energy approximately matches the ratio between $c=1.25$ and $c_{g}=4$ used in this paper. ${ }^{7}$ The remaining parameters are chosen arbitrarily.

\section{The First-Best Scenario}

In this section, we consider the first-best scenario. We assume that the social planner chooses the time path of extraction $q(t)$ and supply of renewable energy $q_{g}(t)$ to maximize the integral of the discounted stream of instantaneous welfare

$$
\int_{0}^{\infty} e^{-r t} W(t) d t
$$

where $W(t)$ is given by (1), subject to $q(t) \geq 0, q_{g}(t) \geq 0, \bar{q}_{g}-q_{g}(t) \geq 0, \dot{S}(t)=-q(t)$, $S(0)=S_{0}, S(t) \geq 0$, and $\dot{X}(t)=\eta q(t), X(0)=X_{0}$.

\subsection{Characterizing the Planner's Solution}

Applying standard optimal control techniques (Leonard and Long 1992), we can show that (see supplementary material, available online) the planner's solution implies a shadow price which can be interpreted as the social cost of carbon. It is given by

$$
\mu(t)=\int_{t}^{\infty} e^{-r(\tau-t)} G^{\prime}(X(\tau)) d \tau
$$

The following assumption will ensure that the resource stock will be exhausted at some finite time:

\footnotetext{
6 In our working paper version (Grondwald et al. 2016), we take account of the heterogeneity of the oil reserves. The results reported in the present paper and in the working paper are broadly the same.

7 In our working paper version, the marginal extraction costs of conventional oils and non-conventional oils are set at $c_{1}=0.75$ and $c_{2}=1.75$. Here we set $c=1.25$; it is an average of $c_{1}$ and $c_{2}$.
} 
Assumption A1 The marginal damage cost when the pollution stock is at its maximum level, $G^{\prime}(\bar{X})$, is small enough so that

$$
\eta \frac{G^{\prime}(\bar{X})}{r}+c<U^{\prime}\left(\bar{q}_{g}\right)
$$

This assumption means that even the last drop of oil has a positive marginal contribution to social welfare. Under Assumption A1, the economy will eventually reach a steady state when the resource stock has been completely exhausted. The steady-state instantaneous welfare level is $\bar{W}=U(\bar{q})-c_{g} \bar{q}-G(\bar{X})$. At the steady state, the social cost of carbon is equal to the present value of the marginal damage flow: $\bar{\mu}=G^{\prime}(\bar{X}) / r$.

At the steady state, the price of energy is $\bar{p} \equiv U^{\prime}\left(\bar{q}_{g}\right)$ while the marginal production cost of clean energy is $c_{g}<U^{\prime}\left(\bar{q}_{g}\right)$. The "profit" flow to the clean energy producers is $\left[\bar{p}-c_{g}\right] \bar{q}_{g}$. This "profit" is the quasi-rent earned by owners of the fixed capacity $\bar{q}_{g}$. Clearly, because $\bar{p}>c_{g}$, it is optimal to use the clean energy source before the exhaustion of the fossil resources.

For the remainder of the paper, we consider a special case of the model: we assume that the damage cost function is linear in the pollution stock: $G(X)=\beta X$ where $\beta>0$. This assumption allows us to have an explicit solution of the model. Then the social cost of carbon is constant over time, $\mu(t)=\beta / r=\bar{\mu}$. Using Hotelling's Rule, at any two dates $t$ and $t^{\prime}$ such the extraction from the stock $S$ is strictly positive, it holds that

$$
[p(t)-(c+\bar{\mu} \eta)] e^{-r t}=\left[p\left(t^{\prime}\right)-(c+\bar{\mu} \eta)\right] e^{-r t^{\prime}}
$$

It follows that the analysis of the three phases of the BAU scenario applies also to the first-best scenario, provided we replace $c$ with $(c+\bar{\mu} \eta)$. For the first-best scenario, the counter-part of Condition 1 is

$$
\text { Condition } 1^{*}: S(0)>S^{*} \equiv \int_{0}^{x^{*}} D\left[c+\bar{\mu} \eta+\left(c_{g}-(c+\bar{\mu} \eta)\right) e^{r \tau}\right] d \tau-x^{*} \bar{q}
$$

where $x^{*}=(1 / r)\left[\ln (\bar{p}-c-\bar{\mu} \eta)-\ln \left(c_{g}-c-\bar{\mu} \eta\right)\right]$. For details, please refer to the online Appendix.

\subsection{Calculation of Welfare in the First-Best Scenario}

Let $t^{*}$ be the time at which the price reaches the production $\operatorname{cost} c_{g}$, so that clean energy begins to be supplied. The social welfare in the first-best scenario is the sum of welfare levels of the three successive phases, $W=W_{1}+W_{2}+W_{3}$, where $W_{i}(i=1,2,3)$ are defined as follows:

$$
\begin{aligned}
& W_{1}=\int_{0}^{t^{*}} e^{-r t}[U(q(t))-c q(t)-\beta X(t)] d t \\
& W_{2}=\int_{t^{*}}^{\bar{T}} e^{-r t}\left[U\left(\bar{q}_{g}+q(t)\right)-c_{g} \bar{q}_{g}-c q(t)-\beta X(t)\right] d t
\end{aligned}
$$

and

$$
W_{3}=e^{-r \bar{T}}\left[\frac{U(\bar{q})-c_{g} \bar{q}_{g}-\beta \bar{X}}{r}\right]
$$


Concerning pollution damages, let us define

$$
\begin{aligned}
& \Omega_{1}=\int_{0}^{t^{*}} e^{-r t}[\beta X(t)] d t, \Omega_{2}=\int_{t^{*}}^{\bar{T}} e^{-r t}[\beta X(t)] d t, \\
& \Omega_{3}=\int_{\bar{T}}^{\infty} e^{-r t}[\beta X(t)] d t .
\end{aligned}
$$

For ease of computation, we assume a quadratic utility function, $U(Q)=A Q-(1 / 2) Q^{2}$. For our base-line scenarion, we set $r=0.01, c=1.25, c_{g}=4, A=20, \bar{q}_{g}=5, S_{0}=2000$ and $X_{0}=100$. Concerning pollution, we assume that $\beta=0.01, \bar{\mu}=\frac{\beta}{r}=1$, and $\eta=1.5$.

We find that phase 1 starts with the initial price $p(0)=3.78$ and ends at time $t^{*}=18.9$, and phase 2 ends at time $\bar{T}=247.14$, with $p(\bar{T})=15$. The welfare calculations reveal that $\sum W_{i}=13,047$ and $\sum \Omega_{i}=1,527$. The ratios of damages to welfare is $11.7 \%$.

\section{Policy Scenario Analysis}

This section moves to the second-best world. We intially describe in more details the so-called Business-as-usual (BAU) scenario-i.e., assuming that there is no government intervention (the carbon tax is zero identically, and there is no subsidy on the clean energy). Next, we build on this situation to analyse the effects of two second best policies: (i) subsidizing the clean energy and (ii) expansion of the clean energy capacity.

\subsection{Sequential Determination of the Key Variables}

We must determine the two endogenous times $t_{g}^{\#}$ and $\bar{T}$, and the initial price $p_{0}$, as functions of the capacity $\bar{q}_{g}$ and the subsidy $s \geq 0$ on the clean energy. The subsidy reduces the firm's unit cost of clean energy from $c_{g}$ to $c_{g}^{\#}(s)=c_{g}-s$. We assume that $c_{g}^{\#}(s)>c$. Recall that $x$ stands for the number of years it would take for the price $p$ to rise from $p=c_{g}$ to $p=\bar{p}$, i.e., $x=(1 / r)\left[\ln (\bar{p}-c)-\ln \left(c_{g}-c\right)\right]$. Let $\widetilde{S}$ denote the required amount of fossil fuel that meets the residual energy demand during the final $x$ years of Phase 2 .

Given $s$, let $t_{g}^{\#}$ be the time at which the price reaches the private cost value $c_{g}^{\#}(s)$. When $s>0$, we have $t_{g}^{\#}<t^{*}$ (the time at which the price reaches the production cost $c_{g}$ ). Thus we have the following relationships

$$
\begin{aligned}
\bar{T} & =t^{*}+(1 / r)\left[\ln (\bar{p}-c)-\ln \left(c_{g}-c\right)\right] \\
t_{g}^{\#} & =t^{*}-h(s)
\end{aligned}
$$

where $h(s)$ is defined as

$$
h(s) \equiv(1 / r)\left[\ln \left(c_{g}-c\right)-\ln \left(c_{g}^{\#}(s)-c\right)\right] .
$$

The cumulative demand for energy from time 0 to the time $t^{*}$, net of the cumulative green energy supplied over the time interval $h(s)$ must equal the initial resource stock minus $\widetilde{S}$ (the quantity of oil needed for the final $x$ years of Phase 2):

$$
\int_{0}^{t^{*}} D(p(t)) d t-\bar{q}_{g} h(s)=S_{0}-\widetilde{S}
$$

where $p(t)=c+\left(c_{g}-c\right) e^{-r\left(t^{*}-t\right)}$. 
The equation

$$
F\left(t^{*}, s, \bar{q}_{g}\right) \equiv \int_{0}^{t^{*}} D(p(t)) d t-\bar{q}_{g} h(s)-S_{0}+\widetilde{S}=0
$$

uniquely determines $t^{*}$ as function of $s$ and $\bar{q}_{g}$. Indeed, we can show that $t^{*}$ increases with $s$ and with the clean energy capacity. After solving for $t^{*}$, we can next determine $\bar{T}$ from $\bar{T}=t^{*}+x$, and $t_{g}^{\#}$ from $t_{g}^{\#}=t^{*}-h(s)$. Then, knowing $\bar{T}$, we use the terminal price $p(\bar{T})$, i.e., $U^{\prime}\left(\bar{q}_{g}\right)$, to determine $p(0)$ and hence all $p(t)$. Next, the stock of pollution at each time $t$ can be computed.

\subsection{Comparative Statics}

This section analyses policy scenarios in which policies aimed at reducing anthropogenic carbon emissions may lead to a Green Paradox.

Following Gerlagh (2011), we say a "weak Green Paradox" is found if there is an increase in current emissions in response to a policy measure whereas a "strong Green Paradox" is associated with an increase in cumulative damages. In our analysis, a weak Green Paradox can be identified as a decrease of $p(0)$, which indicates higher initial resource extraction. Moreover, in this paper we propose the new concept of an extreme Green Paradox, which describes the situation where welfare decreases as a result of misguided (though well-intentioned) climate policy. Our numerical calculations show the existence of extreme Green Paradox outcomes.

To assess the possibility of a Green Paradox, we apply the implicit function theorem the key Eqs. (5), (6), (7) and (8) to determine the response of the endogenous variables $t^{*}, t_{g}^{\#}$ and $\bar{T}$ as well as of price behavior, to changes in the exogenous parameters reflecting the two policy scenarios. Subsidizing the backstop technology is captured by an increase in $s$ whereas an increase in $\bar{q}_{g}$ (which implies a decrease in $\bar{p}$ ) reflects the exogenous increase in capacity.

\subsubsection{Effect of a Subsidy for Renewable Energy}

In the first part of our comparative static analysis, we investigate how subsidizing clean energy affects the extraction speed of the exhaustible resources. It is well known that a subsidy can have detrimental effects on the environment if the clean energy is available at a constant cost without capacity constraint (Strand 2007; Hoel 2011). Our paper, however, assumes that the backstop technology is capacity constrained. Various examples for such subsidy systems exist: the renewable energy feed-in tariffs in Germany and Sweden or the exemption of biofuels from taxation, to name just two. The following proposition summarises the effect of a change in $s$ on the endogenous variables; see the "Appendix" for details.

Proposition 1 An increase in the subsidy rate s for the clean energy output results in (i) a lower initial price $p(0)$, (ii) an earlier time at which the clean energy begins to be produced, i.e., a decrease in $t_{g}^{\#}$, (iii) a delay in the time at which the price $p$ reaches the production cost $c_{g}$, i.e., an increase in $t^{*}$, and (iv) a delay in the date of resource exhaustion, $\bar{T}$. The decrease in $p(0)$ indicates a weak Green Paradox outcome.

\subsubsection{Effect of an Increase in Capacity}

We now investigate the effect of an increase in capacity $\bar{q}_{3}$. This can occur as a result of a technological innovation such as the introduction of electricity storage which allows 
Table 1 Summary of numerical results

\begin{tabular}{lllll}
\hline & First-best & BAU & Subsidy scenario & Capacity increase \\
\hline $\bar{T}$ & 247.14 & 217.01 & 227.74 & 216.03 \\
$t_{g}^{\#}$ & 18.9 & 53.4 & 21.23 & 62.5 \\
$\mathrm{p}(0)$ & 3.78 & 2.82 & 2.66 & 2.72 \\
$\sum \Omega_{i}$ & 1527 & 1712 & 1611 & 1753 \\
$\sum W_{i}$ & 13,047 & 12,973 & 13,012 & 13,174 \\
$\sum_{i} \Omega_{i}$ & 0.11 & 0.13 & 0.12 & 0.13 \\
\hline$\sum_{i} W_{i}$ & & & & \\
\hline
\end{tabular}

massive expansions of wind generating capacities or a move from first-generation to secondgeneration biofuels. An increase in capacity is equivalent to a decrease in the capacity-induced choke price $(\bar{p})$.

Proposition 2 An increase in capacity will (i) lower the initial price $p(0)$, (ii) lower the terminal price $\bar{p}$, (iii) postpone the date at which the capacity will be used (the time at which p reaches $c_{g}$ ), (iv) shorten the phase of simultaneous supply of clean energy and dirty energy. However, the effect on the date of resource exhaustion is ambiguous.

Having completed the analytical exercise, the following section now presents a numerical analysis. This will further illustrate the usefulness of our model and some more refined results will be derived. Furthermore, we conduct a welfare analysis of the second-best policies.

\subsection{Numerical Calculation for the Business-As-Usual Scenario}

The analysis is the same as in the first-best case, except the carbon tax is zero instead of $\bar{\mu}$. We find that $t_{g}^{\#}=53.4$ and $\bar{T}=217.01$. It follows that $p(0)=2.82$. Welfare and damages are reported in Table 1.

Notice that the total damage cost in the BAU scenario is 1712 while under the first-best policy scenario the figure is 1527 . Thus the first-best climate policy reduces total damage cost by about $12 \%$.

\subsection{A Politically Feasible Scenario: Subsidy on Clean Energy}

Now suppose that the government cannot introduce the carbon tax. Instead, suppose the government introduces a subsidy $s=1$ per unit of clean energy, without affecting the capacity. Then clean energy will be produced as soon as the price of oil reaches $p=3$ (instead of 4 as under the BAU scenario). This represents a subsidy rate of $25 \%$. The results are reported in Table 1.

Total welfare under the subsidy is 13,012 . This can be compared to the first best welfare of 13, 047 and the BAU welfare of 12, 973, indicating that the subsidy on clean energy raises welfare above the BAU welfare by about $0.3 \%$. Thus, for our base-line parameter values $(\bar{q}=5$ and $\beta=0.01)$, there is no strong Green Paradox. ${ }^{8}$ The welfare gains relative to the BAU scenario is largely driven by bringing the date of clean energy production much closer

\footnotetext{
8 As a referee suggests, if the green energy capacity is much larger, so that the capacity constraint is "almost non-binding", almost all extraction would be moved closer to the present due to the subsidy, a strong Green Paradox could arise. In Table 2 , we consider very large capacities, $\bar{q}_{g}=15$ and 15.9 , and are able to numerically confirm the result suggested by the referee.
} 
to the present ( $t_{g}^{\#}$ is now 21.23 instead of 53.4), delaying the emissions from a substantial part of the deposit. In fact, the total damage cost under the subsidy scenario is 1611 . Thus the subsidy policy reduces total damage cost by about $5.9 \%$.

\subsection{Politically Feasible Scenario 2: Capacity Increase}

The policy of increasing capacity is very different from a subsidy. If the correct carbon tax is in place, one would not want to use a subsidy. In constrast, in the case of capacity expansion, even if one has the correct carbon tax, it will be welfare increasing to increase capacity if the cost of capacity investment is sufficiently low. In what follows, we consider an increase in capacity from $\bar{q}_{g}=5$ to $\bar{q}_{g}^{\prime}=6$. This represents a capacity expansion of $20 \%$. Assume that this involves an investment cost, denoted by $K$.

The results are reported in Table 1 . With the greater capacity, the constant price that prevails in Phase 3 is now $\bar{p}=U^{\prime}(6)=14$. This lower 'peak' price for oil implies a lower initial price, i.e. $p(0)=2.72$ instead of 2.82 . As a result, under the capacity expansion scenario, there is a short-term increase in extraction and hence short-term increase in damage costs. This is a weak Green Paradox result. We will see below that there is also a strong Green Paradox in this case.

Total welfare (before subtracting $K$, the cost of the capacity expansion) under the capacity expansion is $\sum_{i} W_{i}=13,174$. Note that given our base-line parameter values $\bar{q}=5$ and $\beta=0.01$, the welfare with capacity expansion is higher than the first-best welfare of 13,047 , the BAU welfare of 12,973 and the subsidy welfare of $13,012 .{ }^{9}$

The total damage cost under the capacity expansion scenario is 1753 . The cumulative damages in this scenario are higher than under the subsidy scenario (1611) and the BAU scenario (1712). Thus, a strong Green Paradox occurs. In terms of the present value of the stream of damage costs, Table 1 shows that a subsidy of $25 \%$ on the clean energy will reduce total damage costs by about $5.9 \%$, while a capacity expansion of $20 \%$ will increase total damage costs by about $2.4 \%$. The reason is that in our model with a binding capacity constraint, a subsidy makes clean energy production profitable at an earlier date, without changing the peak price of oil. These effects result in pushing the fossil resource exhaustion date further into the future, so that the pollution stock peaks at a later date, $\bar{T}=227.74$. In contrast, a capacity expansion reduces the maximum price that the last drop of oil would earn ( $\bar{p}$ falls from 5 to 4 ). This results in a strong incentive for fossil resource owners to increase their earlier extraction.

\section{Sensitivity Analysis}

\subsection{Sensitivity with Respect to the Damage Parameter}

Since there is great uncertainty about the magnitude of potential damages associate with carbon emissions, it is important to explore the sensitivity of our results to changes in the damage parameter $\beta$. Recall that in our benchmark simulation, we set $\beta=0.01$. In this subsection we report the effects of considering two additional parameter settings: $\beta=0.02$ as well as $\beta=0.08$. Please note that this will result in proportional changes in the damage costs in all scenarios except for the first-best case, where the carbon tax will be optimally

\footnotetext{
9 A referee points out that if $\beta$ is sufficiently high, the Green Paradox effect will be so strong that welfare (under the capacity expansion scenario) would decline (relative to BAU). We will report such a numerical result in Table 2, thus confirming the referee's point.
} 


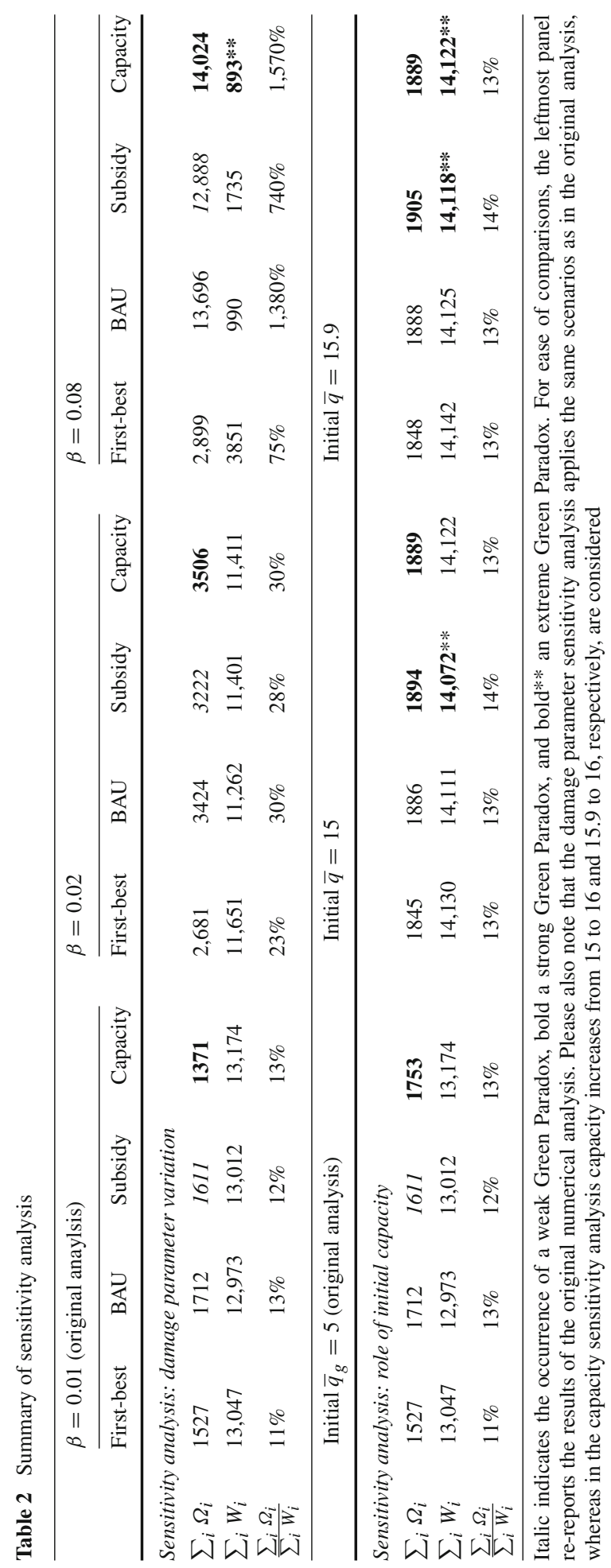


adjusted, implying that the damage costs will increase by a smaller percentage. The upper panel of Table 2 presents the results.

Among the noteworthy results we should mention a few comparisons. First, the welfare effects of the subsidy are, for $\beta=0.02$, not much different from those of the capacity increase; for $\beta=0.01$ the difference was substantial. Second, for both the subsidy and the capacity increase, now negative welfare effects are found compared to the first-best case. For $\beta=0.08$ the Green Paradox effect associated with the capacity expansion is so strong that the welfare is even lower than in the BAU scenario. We refer to this as an "extreme Green Paradox" outcome. ${ }^{10}$

\subsection{Sensitivity with Respect to the Capacity Constraint}

In the benchmark case, we assume that green energy capacity is 5, and thus the steady state price is 15 . It is worth studying as well the case where the capacity constraint is almost nonbinding. We consider two alternative initial capacity levels: $\bar{q}_{g}=15$ as well as the extreme case, $\bar{q}_{g}=15.9$ (and the steady state prices are $\bar{p}=5$ and $\bar{p}=4.1$ respectively). Note that we continue to assume that $\beta=0.01$. The lower panel of Table 2 reports the results.

For $\bar{q}_{g}=15$ and hence $\bar{p}=5$, we find that, under the first-best carbon tax, the damage costs are higher than in the original low capacity case. This is because the high capacity implies a lower peak price for the resource, $\bar{p}=5$ (instead of 15), and thus encourages earlier exhaustion. In addition, the difference between the damage costs under the first-best and BAU is smaller than in the low capacity case. The total damages under the subsidy scenario are slightly smaller than under BAU. At this large capacity of $\bar{q}_{g}=15$, the subsidy gives rise to a strong Green Paradox. The capacity expansion policy (increasing $\bar{q}_{g}$ from 15 to 16) creates an increase in damages compared with BAU. Notice that with such an expansion, the capacity constraint is no longer binding: $\bar{p}$ falls from 5 to 4 , which is equal to the marginal $\cos t c_{g}$ of the clean energy. At that price, demand is exactly equal to the expanded capacity, 16. As a result, resources are exhausted early on, bringing damages closer to the present. Welfare under capacity expansion is however still higher than under BAU, because the steady state price under BAU is $\bar{p}=5$ while that under capacity expansion is $\bar{p}=4$.

For $\bar{q}_{g}=15.9$ and hence $\bar{p}=4.1$, we consider the effects of a capacity expansion to $\bar{q}_{g}=16$, or of a subsidy $s=1$. Under the first-best carbon tax, we find that both welfare and damages are higher than the corresponding values for $\bar{q}=15$. Compared with BAU, the damages under the subsidy are higher and the welfare under the subsidy is lower; thus, there are a strong Green Paradox (in terms of damages) and an extreme Green Paradox (in terms of welfare). Concerning the effects of a capacity expansion, we find that welfare under BAU is higher than under capacity expansion, which is an extreme Green Paradox.

\section{Illustration of Policy Relevance}

The model presented in this paper exhibits a considerable degree of flexibility and is able to capture various empirical observations as well as challenges policy makers currently face. To illustrate this wide applicability, this section provides (stylized) evidence that supports this paper's approach, showing that it is highly relevant. In addition to the crude oil market application introduced in Sect. 2 and analysed in detail in Sect. 4, this section illustrates

10 We are grateful to an anonymous referee for encouraging us to consider this possibility. 
additional applications for this paper's model for the analysis of the transformation of the electricity sector.

As already mentioned above, the model presented in this paper can be easily extended to allow applications to an oil market with conventional and unconventional oil as well as biofuels as a clean substitute. The parametrisation of such an extended model (as in Grondwald et al. 2016) generally reflects the cost structure and environmental impacts in this sector. The consideration of two rather than one "dirty" resource would allow us to capture unconventional carbon resources such as extra heavy oil, oil sands, and oil shale; see Gordon (2012). Extracting oil from unconventional sites is more costly as well as more energy intensive and, thus, unconventional oil has a higher $\mathrm{CO}_{2}$ emission intensity and extraction cost than conventional oil. Specifically, in addition to various technological problems, biofuel production raises land use concerns as probably there will not be enough (suitable) land available for biofuel production and, even if there were, using it for that purpose might seriously compromise food production and raise sustainability concerns; see, e.g., Sinn (2012). Thus, it seems plausible to assume that there is a constraint imposed on the share of biofuels production. The share of biomass from global primary energy supply is currently about $15 \%$. This, however, is to a very large extent attributable to so-called "traditional biomass"-the use of firewood, charcoal as well as agricultural residues; see International Energy Agency (2012). The share of biofuels in global road transport, however, is merely 3\% and several problems indicate that it is more than reasonable to assume that biomass is not a backstop technology that can be used without constraints; see International Energy Agency (2011). ${ }^{11}$ Our model not just allows us to capture this issue, it is furthermore possible to analyse the effect of changes in this capacity. In light of the finding of possible negative welfare effects under the capacity expansion scenario, the global biomass potential that actually exists would have to be seen as a considerable problem.

Applications of our model are not restricted to the crude oil market: the electricity market is another possible application for our model. The overall situation there is a similar to the oil market example: Electricity is generated from both different "dirty" and exhaustible conventional resources as well as clean ones simultaneously - despite the fact that renewable energy is considerably more expensive than conventionally produced electricity. In order to fight climate change, decrease the dependency on imports of energy resources as well as the issue of resource scarcity contribute to the attractiveness of renewable energies. In consequence, wind or solar power is used instead of (or at least in addition to) coal or gas. As a result, policy instruments such as feed-in-tariffs or clean energy quotas are in place in many countries. For example, Germany today generates approximately $20 \%$ of total electricity from renewable sources such as wind and solar and the European Union aims at reaching this share at the European level by 2020 . However, further increasing this share seems to be more challenging than originally expected. For example, substantial investments into the electricity transmission and distribution network are required. What is more, the problems of intermittent renewable energies and the considerable lack of storage facilities are still unresolved. In addition to these technological challenges, there are also important regulatory ones. The requirement of backup power plants to guarantee network stability sparked the debate on an entire redesign of electricity market-the introduction of so-called capacity

11 Even though projections certainly indicate that there is a vast potential for biomass (for example, according to International Energy Agency (2011), unused and surplus land has the potential of about 550-1500 EJ biomass production in 2050), the way to exploit this potential is nevertheless long and stony. To mention just a few of the challenges, crop yields need to increase considerably, and substantial parts of land needs to be converted. In addition to that, International Energy Agency (2011) points to regulatory requirements and stresses the importance of ensuring that food security is not compromised; see also Sinn (2012). 
markets is among the options. Finally, the requirements of the politically important so-called triangle of energy supply - energy is supposed to be sustainable, affordable, and reliableeffectively constrain the further development of renewable energies in electricity production. In short, assuming that a backstop resource for electricity generation is unconstrained is highly unrealistic. In light of our findings policy instruments aiming at an increase of the capacity constraint of a renewable substitute are problematic. However, subsidizing this technology and, thus, developing it to market maturity earlier may have positive long-term effects. However, a detailed analysis of possible Green Paradox effects in the electricity market requires a corresponding calibration of the numerical model.

Finally, our model is also be able to capture the issue of nuclear energy. This "conventional," but carbon-free form of energy is constrained by regulatory, political, and maybe even (safety-related) technological restrictions. ${ }^{12}$

These reflections vividly illustrate the wide applicability of this paper's model. It is fairly obvious that applications of this model make an important contribution to current energy policy debates. In a nutshell, the model applied in this paper is able to capture various empirical energy market observations and the results obtained in this paper clearly indicate that ignoring the important feature of capacity-constrained backstop technologies can lead to inappropriate policy recommendations.

\section{Conclusions}

It is no exaggeration to state that climate change is among the biggest challenges mankind has ever been faced. Thus, it is very important that we respond appropriately to this challenge. Perhaps for this very reason and perhaps just because we need to respond soon, this challenge is particularly difficult. Economic analyses identified various well-intentioned climate policy measures in the past which, at first glance, appeared useful, but after taking a closer look, turned out to be counterproductive. While this literature has a long historyolder contributions date back to the 1980s and 1990s-there is a recent stream of literature sparked by Sinn's (2008) discovery of the so-called Green Paradox. The basic finding of that paper is that the owners of exhaustible fossil resources possibly bring forward extraction of their resources as a response to intensifying climate policies. Because of the importance of finding an appropriate answer, however, it is also necessary to use appropriate economic modelling frameworks. If these frameworks are not designed carefully enough there is a risk that inappropriate policy recommendations emerge from these research efforts.

Sinn's (2008) original findings are very elegantly derived and are also intuitively very convincing. However, in response to Sinn's paper, a large number of papers emerged which can be summarized as follows: the more realistic the modelling approach is the more detailed the results become. This paper's findings fit very well into this overall landscape. The model used here allows to capture two important empirical observations. First, even though clean technologies are generally available-wind and solar energy seem to be suitable for replacing coal and gas power plants, cars could very well be run on biofuels rather than conventional fuels, clean and dirty technologies are used simultaneously. Second, further expanding and implementing clean technologies increasingly meets resistance. In more and more countries, in particular Germany and the United Kingdom, there are significant local

\footnotetext{
12 Finally even the assumption that the constrained backstop technology is clean could be relaxed. The case of a dirty backstop technology is studied in van der Ploeg and Withagen (2012a). Liquid fuels produced with coal-to-liquids technologies serve as one example for a dirty but certainly also constrained backstop technology.
} 
initiatives to oppose the installation of additional wind parks. Extending the use of biofuels is a major concern for organisations which care about food security and food prices. Thus, there is sufficient evidence to assume that the use of clean technologies is constrained. These very constraints are most likely the reasons why different types of energy sources are used simultaneously even though costs associated with their use differ dramatically: solar energy is still much more expensive than conventional electricity, to name just one example.

As currently existing models are not able to capture these two observations, this paper makes an important contribution to this literature. As stated above, it is important to use appropriate economic models in order to avoid the possibility of deriving inappropriate policy recommendations. In addition, our model allows for the analysis of an entirely new scenario: the expansion of the capacity constrained clean energy. The results indicate that this scenario is considerable more harmful than a subsidization of the clean energy. In addition, we show that the initial capacity size is crucial for the effects of the policy scenarios.

Various channels through which a Green Paradox can occur have been discussed in the literature: intertemporal arbitrage, spatial, technological, or extraction order effects; see van der Ploeg and Withagen (2015) and Jensen et al. (2015). Intertemporal effects play a large role in Sinn's (2008) paper as well as in the earlier contribution by Long and Sinn (1985). A technology-induced Green Paradox has been identified by Strand (2007). In this paper the intertemporal channel is important but also the capacity constraint plays an important role. The theoretical framework used in this paper is based on Holland's (2003) analysis of extraction capacities and the optimal order of extraction of exhaustible resources. This model is re-interpreted and considerably extended to include emissions and pollution damages. In order to operationalize the concept of the Green Paradox in greater detail, this paper, in addition, borrows from Gerlagh (2011) and considers different degrees of the Green Paradox. In addition, the paper also introduces the concept of an extreme Green Paradox.

What is fascinating to observe is that a large number of papers emerged only in response to Sinn's (2008) discovery of the Green Paradox. The theoretical framework used in many if these papers is almost 100 years old and goes back to Hotelling (1930). For a surprisingly long period this literature remained is a hibernation-type state. The oil price hike in the 1970s provided impetus for deepening the Hotelling framework. In the 1990s, a number of papers addressed the issue of global warming. In terms of climate implications of feasible secondbest policies, the big awakening came with Sinn (2008). What we can now hope is that the concerted research efforts that paper sparked helps identify the responses we need to apply if we want to keep climate change under control.

\section{Compliance with Ethical Standards}

Conflicts of interest The authors declare that they have no conflict of interest.

Open Access This article is distributed under the terms of the Creative Commons Attribution 4.0 International License (http://creativecommons.org/licenses/by/4.0/), which permits unrestricted use, distribution, and reproduction in any medium, provided you give appropriate credit to the original author(s) and the source, provide a link to the Creative Commons license, and indicate if changes were made. 


\section{Appendix}

\section{Derivations of Comparative Results}

The effect of a change in $s$ and $\bar{q}_{g}$ (or, equivalently, in $\bar{p}$ ) on the endogenous variables can be computed as follows. Using Eq. (8), we get

$$
\frac{\partial t^{*}}{\partial s}=-\frac{F_{s}}{F_{t}^{*}}>0 \quad \text { and } \quad \frac{\partial t^{*}}{\partial \bar{q}_{g}}=-\frac{F_{\bar{q}_{g}}}{F_{t^{*}}}>0
$$

where

$$
\begin{aligned}
F_{t^{*}} & =D\left(c_{g}\right)-r\left(c_{g}-c\right) \int_{0}^{t^{*}} D^{\prime}(p(t)) e^{-r\left(t^{*}-t\right)} d t>0 \\
F_{s} & =-\bar{q}_{g} \frac{1}{r\left(c_{g}^{\#}(s)-c\right)}<0 \\
F_{\bar{q}_{g}} & =-\frac{1}{r} \ln \left(\frac{c_{g}-c}{c_{g}^{\#}(s)-c}\right)+\frac{d \widetilde{S}\left(\bar{q}_{g}\right)}{d \bar{q}_{g}}=-\frac{1}{r} \ln \left(\frac{c_{g}-c}{c_{g}^{\#}(s)-c}\right)-x \\
& =-\frac{1}{r} \ln \left(\frac{c_{g}-c}{c_{g}^{\#}(s)-c}\right)-(1 / r)\left[\ln \left(U^{\prime}\left(\bar{q}_{g}\right)-c\right)-\ln \left(c_{g}-c\right)\right] \\
& =\frac{1}{r} \ln \left(c_{g}^{\#}(s)-c\right)-\frac{1}{r} \ln \left(U^{\prime}\left(\bar{q}_{g}\right)-c\right)=-x_{g}^{\#}<0
\end{aligned}
$$

Using the Hotelling Rule, $p(0)=c+\left(c_{g}-c\right) e^{-r t^{*}}$, we obtain

$$
\frac{d p(0)}{d s}=-e^{-r t^{*}}\left(c_{g}-c\right) \frac{d t^{*}}{d s}<0
$$

Next, consider the effect of a subsidy on the length of Phase $1, t_{g}^{\#}$. Using

$$
t_{g}^{\#}=t^{*}-h(s)=t^{*}+\frac{1}{r} \ln \left[\frac{c_{g}^{\#}(s)-c}{c_{g}-c}\right]
$$

we obtain

$$
\begin{aligned}
\frac{d t_{g}^{\#}}{d s} & =\frac{d t^{*}}{d s}-\frac{1}{r\left(c_{g}^{\#}(s)-c\right)} \\
& =\frac{1}{r\left(c_{g}^{\#}(s)-c\right)}\left\{\frac{\bar{q}_{g}}{D\left(c_{g}\right)-r\left(c_{g}-c\right) \int_{0}^{t^{*}} D^{\prime}(p(t)) e^{-r\left(t^{*}-t\right)} d t}-1\right\}<0
\end{aligned}
$$


The effects of a capacity expansion are computed as follows.

$$
\begin{aligned}
\frac{d t^{*}}{d \bar{q}_{g}} & =-\frac{\frac{1}{r} \ln \left(c_{g}^{\#}(s)-c\right)-\frac{1}{r} \ln \left(U^{\prime}\left(\bar{q}_{g}\right)-c\right)}{D\left(c_{g}\right)-r\left(c_{g}-c\right) \int_{0}^{t^{*}} D^{\prime}(p(t)) e^{-r\left(t^{*}-t\right)} d t}>0 \\
\frac{d t_{g}^{\#}}{d \bar{q}_{g}} & =\frac{d t^{*}}{d \bar{q}_{g}}-\frac{d h}{d \bar{q}_{g}}=\frac{d t^{*}}{d \bar{q}_{g}}>0 \\
\frac{d p(0)}{d \bar{q}_{g}} & =-e^{-r t^{*}}\left(c_{g}-c\right) \frac{d t^{*}}{d \bar{q}_{g}}<0 \\
\frac{d \bar{T}}{d \bar{q}_{g}} & =\frac{d t^{*}}{d \bar{q}_{g}}+\frac{d x}{d \bar{q}_{g}} \\
& =-\frac{\frac{1}{r} \ln \left(c_{g}^{\#}(s)-c\right)-\frac{1}{r} \ln \left(U^{\prime}\left(\bar{q}_{g}\right)-c\right)}{D\left(c_{g}\right)-r\left(c_{g}-c\right) \int_{0}^{t^{*}} D^{\prime}(p(t)) e^{-r\left(t^{*}-t\right)} d t}+\frac{U^{\prime \prime}\left(\bar{q}_{g}\right)}{r\left(U^{\prime}\left(\bar{q}_{g}\right)-c\right)} \lessgtr 0
\end{aligned}
$$

\section{References}

Dasgupta P, Heal G (1974) The optimal depletion of exhaustible resources. Rev Econ Stud 41:3-28. Symposium on the economics of exhaustible resources

Gerlagh R (2011) Too much oil. CESifo Econ Stud 57(1):79-102

Gordon D (2012). Understanding unconventional oil. The Carnegie papers

Grafton Q, Kompas T, Long NV (2012) Substitution between biofuels and fossil fuels: is there a Green Paradox? J Environ Econ Manag 64(3):328-341

Grondwald M, Long NV, Roepke L (2016) Simultaneous supplies of dirty energy and capacity constrained clean energy: is there a Green Paradox? Working paper 2016-s-61, CIRANO, Montreal

Hoel M (2011) The supply side of $\mathrm{CO}_{2}$ with country heterogeneity. Scand J Econ 113(4):846-865

Hoel M, Jensen S (2012) Cutting costs of catching carbon-intertemporal effects under imperfect climate policy. Resour Energy Econ 34:680-695

Holland S (2003) Extraction capacity and the optimal order of extraction. J Environ Econ Manag 45(3):569588

Hotelling H (1930) The economics of exhaustible resources. J Polit Econ 39(2):137-175

International Energy Agency (2011) Technology roadmap-biofuels for transport. International Energy Agency, Paris

International Energy Agency (2012) World energy outlook 2012. International Energy Agency, Paris

Jensen S, Mohlin K, Pittel K, Sterner T (2015) An introduction to the green paradox: the unintended consequences of climate policies. Rev Environ Econ Policy 9(2):246-265

Leonard D, Long NV (1992) Optimal control theory and static optimization in economics. Cambridge University Press, Cambridge

Long NV, Sinn H-W (1985) Surprise price shift, tax changes and the supply behaviour of resource extracting firms. Aust Econ Pap 24(45):278-289

Michielsen TO (2014) Brown backstops versus the green paradox. J Environ Econ Manag 68:87-110

Sinn H-W (2008) Public policies against global warming: a supply side approach. Int Tax Public Finance 15(4):360-394

Sinn H-W (2012) The Green Paradox: a supply-side approach to global warming. MIT Press, Cambridge, MA Strand J (2007) Technology treaties and fossil fuels extraction. Energy J 28:129-142

van der Ploeg F, Withagen C (2012a) Too much coal, too little oil. J Public Econ 96(1):62-77

van der Ploeg F, Withagen C (2012b) Is there really a Green Paradox? J Environ Econ Manag 64(3):342-363 van der Ploeg F, Withagen C (2015) Global warming and the green paradox: a review of adverse effects of climate policies. Rev Environ Econ Policy 9(2):285-303 